\title{
A Chronic Scheme of Cranial Window Preparation to Study Pial Vascular Reactivity in Murine Cerebral Malaria
}

\author{
PENG KAI ONG, * DIANA MEAYS, * JOHN A. FRANGOS, * AND LEONARDO J.M. CARVALHO* $\dagger$ \\ ${ }^{*}$ Center for Malaria Research, La Jolla Bioengineering Institute, San Diego, California USA,; ${ }^{\dagger}$ Laboratory of Malaria Research, Oswaldo Cruz Institute, \\ Rio de Janeiro, Brazil \\ Address for correspondence: Peng Kai Ong, Ph.D., Center for Malaria Research, La Jolla Bioengineering Institute, 3535 General Atomics Court suite 210, \\ San Diego, CA 92121, USA. E-mail: pkong@ljbi.org
}

Received 29 October 2012; accepted 14 December 2012.

\begin{abstract}
Objective: The acute implantation of a cranial window for studying cerebroarteriolar reactivity in living animals involves a highly surgically invasive craniotomy procedure at the time of experimentation, which limits its application in severely ill animals such as in the experimental murine model of cerebral malaria (ECM). To overcome this problem, a chronic window implantation scheme was designed and implemented.

Methods: A partial craniotomy is first performed by creating a skull bone flap in the healthy mice, which are then left to recover for one to two weeks, followed by infection to induce ECM. Uninfected animals are utilized as control. When cranial superfusion is needed, the bone flap is retracted and window implantation completed by assembling a perfusion chamber for compound delivery to the exposed brain surface. The presurgical step is intended to minimize surgical trauma on the day of experimentation.

Results: Chronic preparations in uninfected mice exhibited remarkably improved stability over acute ones by significantly reducing periarteriolar tissue damage and enhancing cerebroarteri-
\end{abstract}

olar dilator responses. The chronic scheme was successfully implemented in ECM mice, which unveiled novel preliminary insights into impaired cerebroarteriolar reactivity and eNOS dysfunction. Conclusion: The chronic scheme presents an innovative approach for advancing our mechanistic understanding on cerebrovascular dysfunction in ECM.

Key words: cerebroarteriolar responses, cranial window superfusion, endothelial nitric oxide synthase, experimental cerebral malaria, Plasmodium berghei ANKA.

Abbreviations used: ACh, acetylcholine; aCSF, artificial cerebrospinal fluid; ECM, experimental cerebral malaria; eNOS, endothelial nitric oxide synthase; GFP, green fluorescent protein; GSNO, $S$-nitroso-L-glutathione; IV, intravascular; L-NMMA, L- $N^{\mathrm{G}}$-monomethyl arginine; MAP, mean arterial blood pressure; $\mathrm{NO}$, nitric oxide; $\mathrm{PbA}$, Plasmodium berghei ANKA; PB, Pacific ${ }^{\mathrm{TM}}$ Blue; PI, propidium iodide; PV, perivascular; $V$, mean cellular velocity; VFR, volume flow rate.

Please cite this paper as: Ong PK, Meays D, Frangos JA, Carvalho LJM. A chronic scheme of cranial window preparation to study pial vascular reactivity in murine cerebral malaria. Microcirculation 20: 394-404, 2013.

\section{INTRODUCTION}

The intrinsic ability of pial arterioles to constrict or dilate in response to their environmental changes in mechanical and biochemical stimuli (cerebrovascular reactivity) is a physiologically important phenomenon as they are key regulators of cerebral blood flow and hence metabolic homeostasis in the brain [12]. The closed cranial window for superfusion is an experimental technique widely utilized for studying cerebrovascular reactivity in living animals. It integrates the microscopic visualization of superficial pial arterioles via a craniotomy with the quantification of their diameter responses to the application of soluble factors delivered through a perfusion chamber assembled on the skull $[15,23]$. By using an assortment of agonistic and/or antagonistic agents to target specific pathways in endothelial cells, neurons and astrocytes linked to downstream induction of changes in the cerebrovascular tone, this technique has been successfully employed in elucidating mechanisms that are responsible for the impairment of cerebrovascular reactivity seen in various cerebrovascular and neurodegenerative diseases (diabetes mellitus [19], hypertension [33], hyperammonemia [14], alcoholism [34], obesity [8], and Alzheimer's disease [26]). A better understanding of participating mechanisms in these pathologies is important to facilitate the development of effective therapeutic targets via restoration of vascular responses $[4,36]$.

Despite its seemingly obvious advantages, a significant drawback in the use of the cranial window method lies in its unappealing surgical invasiveness in the procedures (skin 
excision and skull removal) for the craniotomy [24]. When combined with an acute scheme which essentially involves performing the surgery at the time when observation of cerebrovascular responses is needed, this technique becomes an unattractive candidate to be applied in scenarios of pathology where neurophysiology and physical well-being of the animal are known to be severely compromised. Therefore, it is perhaps unsurprising that conventional experiments on cranial superfusion have been confined to studies whereby pathogenesis or induction of the disease is minimally deleterious to the physical health of the animal at the time of study [33]. Indeed, the cranial window scheme for superfusion is well recognized as a valuable source of information when conducted through means that do not or minimally affect cardiophysiology, for instance, the use of transgenic animals with the desired target gene mutations $[21,25]$. Nevertheless, the surgical intervention can still bring about other undesirable consequences: increased susceptibility to loss of vascular responses accompanying vascular damage due to frictional heat generated during the skull drilling procedure [15] as well as induction of an inflammatory immune response that can potentially interfere with the experimental outcome [39].

A new technique that can overcome the aforementioned limitations associated with the acute cranial window scheme is urgently needed to study the cerebral microvascular effects of malaria infection. The murine model of cerebral malaria by Plasmodium berghei ANKA infection is an extensively used animal model for studying CM pathogenesis as it shares many similar pathological features with human CM [22]. Murine CM or ECM is associated with a vasculopathy characterized by cerebral arteriolar constriction and reduced cerebral blood flow [3]. Although this seems to suggest an alteration in cerebrovascular tone during ECM pathogenesis, cerebrovascular reactivity in ECM is poorly understood and remains to be elucidated. It may be alluring to take advantage of the murine cranial window scheme as a research tool to tackle this problem. However, ECM development based on the murine model, which typically occurs between the fifth and the tenth day of infection, is characterized by rapid deterioration of the animal's physical condition once clinical signs (such as ataxia, impaired locomotion and hypothermia) became apparent [6] . Furthermore, a loss of blood clotting factors, which could lead to profuse bleeding at the surgical site [31] coupled with the delicate nature of the brain tissue complicated by tissue edema and petechial hemorrhages formation [17], would preclude one from applying the highly surgically invasive acute scheme in a murine ECM setting. Indeed, our preliminary studies on the application of the acute scheme in ECM were met with little success, being plagued by the above problems.

To overcome limitations associated with the acute scheme, we introduced a new chronic scheme of the cranial window technique for superfusion suitable for investigating pial arteriolar reactivity in $\mathrm{PbA}$-infected $\mathrm{C} 57 \mathrm{BL} / 6$ mice with ECM. In this scheme, the major surgical procedures (skin removal and skull drilling) are performed in the healthy animals, which are adequately recovered followed by infection to induce ECM, where necessary. The cranial window implantation is subsequently carried out in animals (uninfected control or ECM mice) when cranial superfusion is required. The rationale here is to minimize surgical intervention on the day of the superfusion experiment, which would help improve preparation stability. We showed that in healthy animals, preparations based on the new scheme exhibited improved stability over those of the acute scheme, and by successfully applying the new scheme in the ECM mice, we further demonstrated its capability of unveiling novel mechanistic insights into ECM pathogenesis.

\section{MATERIALS AND METHODS}

\section{Presurgical Procedure}

Animal handling and care followed the National Institutes of Health Guide for Care and Use of Laboratory Animals. All protocols were approved by the La Jolla Bioengineering Institutional Animal Care and Use committee. Eight-weekold female C57BL/6 mice (Jackson Laboratories, Bar Harbor, ME, USA) weighing 18-22 g were anesthetized with intraperitoneal administration of ketamine $(150 \mathrm{mg} / \mathrm{kg})$ and xylazine $(10 \mathrm{mg} / \mathrm{kg})$. Dexamethasone $(0.2 \mathrm{mg} / \mathrm{kg})$, carprofen $(5 \mathrm{mg} / \mathrm{kg})$ and ampicillin $(6 \mathrm{mg} / \mathrm{kg})$ were injected subcutaneously to prevent brain swelling, inflammation, and bacterial infection, respectively $[11,24]$. Full sedation of the animal was ensured during the surgery by repeatedly testing the animal reflex responses to toe/tail pinches. The initial surgical steps here followed closely those typically used in the preparation of a craniotomy for an open-skulled cranial window assembly [24] until the point of creating a bone flap for skull removal. At this stage, the earlier drilling along an $\sim 3 \mathrm{~mm}$-diameter circular outline on the right parietal skull bone had produced a loose bone flap (Figure 1A). Instead of retracting the bone flap, it was covered with low temperature-melt bone wax, while the remaining skull area was filled with dental acrylic (Figure 1B). It is important in this step to ensure that the skull surface is dry to allow optimal adhesion of the bone wax and the acrylic for long-lasting protection. After recovery from surgery, the mice were given drugs (carprofen and ampicillin) daily for five days. Animals presenting signs of pain or discomfort were euthanized by intraperitoneal injection of euthasol $(100 \mathrm{mg} / \mathrm{kg})$.

Mice were randomly assigned to the uninfected and infected groups. In the uninfected group, animals were left to recover for two weeks before implementing the chamber assembly step. This serves as a time control for arteriolar responses in the infected animals to ensure that any alteration in responses observed during infection is not a time-dependent 

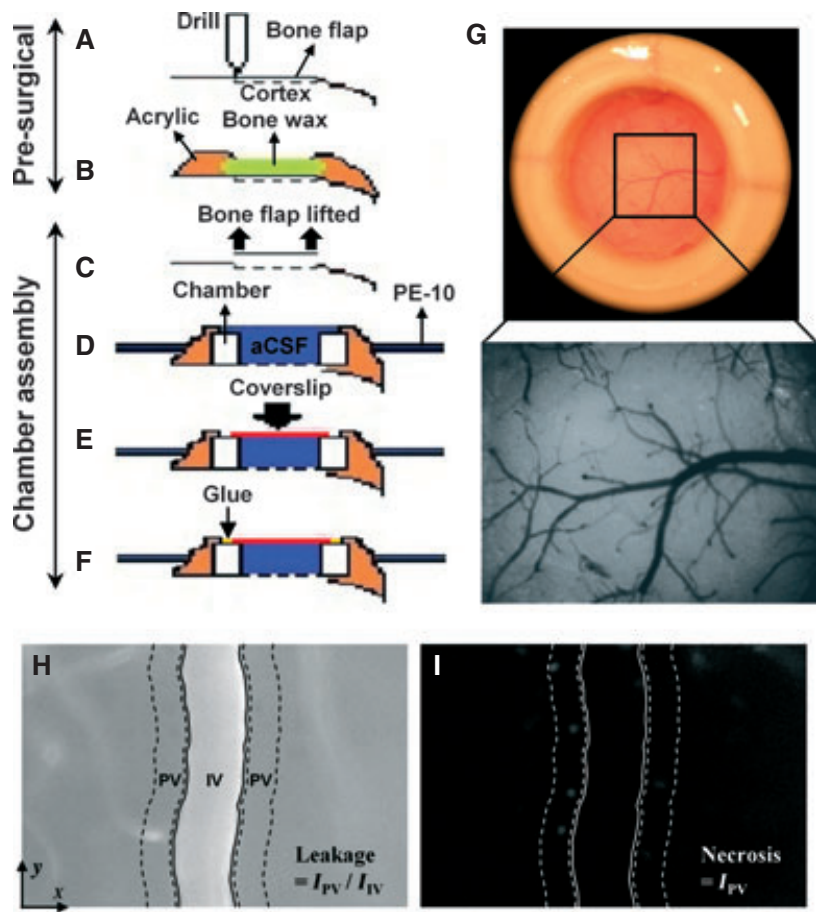

Figure 1. A-F: Key steps in window preparation based on the chronic scheme. Presurgical procedure: Skull drilled to create bone flap. (A) Bone flap covered with bone wax and the remaining skull area filled with dental acrylic. (B) Chamber assembly for superfusion after mouse recovery and/or infection: Bone flap retracted following removal of bone wax. (C) Chamber fixed on skull and filled with aCSF with exposed cortex positioned at its internal opening. (D) Chamber top sealed with glued-on coverslip (E and F). (G) Completed window assembly and visualization of the pial microcirculation through window. ( $\mathbf{H}$ and $\mathbf{I})$ Image analyses for quantification of vascular leakage and tissue necrosis based on vessel images stained by albumin-FITC and PI, respectively. $I_{\mathrm{PV}}$ and $I_{\mathrm{IV}}$ : Mean intensities of PV and IV regions, respectively. Boundaries of the PV region (dashed lines) on each side of the vessel were defined at distances of $2.6 \mu \mathrm{m}$ (assumed endothelium width) and $0.5 \times$ of vessel diameter from the wall outline (solid line).

phenomenon that is occurring even in the absence of infection. Mice in the infected group were allowed to recover for one week, following which $10^{6} \mathrm{PbA}$ parasites expressing GFP (PbA-GFP, a donation from the Malaria Research and Reference Reagent Resource Center-MR4, Manassas, VA; deposited by CJ Janse and AP Waters; MR4 number: MRA$865)$ were intraperitoneally inoculated. Chamber assembly in these animals was carried out on day 5-6 of infection after they had developed clinical signs of ECM accompanied by hypothermia and low motor behavioral score.

\section{Chamber Assembly Step}

The chamber assembly step was initiated by anesthetizing the mouse using isofluorane ( $4 \%$ for induction, $1-2 \%$ for maintenance). After ensuring that the animal was not responding to any reflex test, it was secured in a prone position under a stereotaxic frame. Using a blunt microsurgical blade, the bone wax was gently scraped off the skull surface, which was then cleaned with several swipes of sterile saline. After that, the bone flap previously created was carefully lifted off under a drop of sterile saline using a pair of forceps with very fine tips to expose the underlying cortex (Figure 1C). Using the sharp tip of a needle (30G), an incision was made in the dura mater, which was then carefully retracted to the edge of the open skull. Any minor bleeding of the exposed cortical surface was quenched using gelfoam soaked previously in sterile aCSF (Harvard Apparatus, Holliston, MA, USA), which has an ionic constitution resembling the brain CSF ( $150 \mathrm{mM} \mathrm{Na}, 3.0 \mathrm{mM} \mathrm{K}, 1.4 \mathrm{mM}$ Ca, $0.8 \mathrm{mM} \mathrm{Mg}, 1.0 \mathrm{mM} \mathrm{P}$; $155 \mathrm{mM} \mathrm{Cl}$ ). A prefabricated chamber consisting of a ring structure (made of polyether ether ketone, with an internal volume of $\sim 10 \mu \mathrm{L}$, equipped with inlet and outlet ports connected to polyethylene tubes (PE-10; Harvard Apparatus) and a third port for intracranial pressure monitoring) was glued (Vetbond ${ }^{\mathrm{TM}} ; 3 \mathrm{M}$, St. Paul, MN, USA) to the skull with an orientation that positions the exposed cortex at the center of its internal opening (Figure 1D). Dental acrylic was applied around the chamber to secure it to the skull and to prevent leakage. The chamber was then filled completely with sterile aCSF through the inlet tube and by taking advantage of the liquid suction force, a glass coverslip was rested on top of the chamber (Figure 1E). The chamber assembly was completed by applying glue around the edge of the coverslip to seal off the top of the chamber (Figure $1 \mathrm{~F}$ ). Figure $1 \mathrm{G}$ shows a completed chronic window assembly in an uninfected mouse with clear visualization of pial vessels through the window. On the other hand, in the case of an acute window preparation, chamber assembly was carried out following the craniotomy on the day of experiment [23].

\section{Intravital Microscopy}

After the chamber assembly step, the isofluorane supply was discontinued and $\alpha$-chloralose (Sigma-Aldrich, St. Louis, MO, USA) was infused intraperitoneally with a loading dose of $30 \mathrm{mg} / \mathrm{kg}$, followed by a maintenance dose of $25 \mathrm{mg} / \mathrm{kg}$ every hour for the remainder of the experiment [29]. The mouse was transferred to an intravital microscopic stage (customized Leica-McBain, San Diego, CA, USA) and its body temperature maintained by a heating pad. The exposed cortical surface was preconditioned with the aCSF by continuously flushing prewarmed aCSF (maintained at $37^{\circ} \mathrm{C}$ and $\mathrm{pH}$ of $7.35-7.45$ by equilibration with a gas mixture of $6.5 \% \mathrm{O} 2,10 \% \mathrm{CO} 2$ and balance $\mathrm{N} 2$ ) through the chamber for 30 minutes, at a flow rate of $10 \mu \mathrm{L} / \mathrm{min}$ with the help of a syringe pump (Pump 33; Harvard Apparatus). During this period, the pial microcirculation was visualized through the cranial window under a drop of water using a $20 \times$ water immersion objective (LUMPFL-WIR, numerical aperture 0.6; Olympus, Center Valley, PA, USA). Arterioles and/or venules for analyses $(n=1-4$, vessel diameter $[D]=20-70 \mu \mathrm{m})$ were randomly selected and spatial 
mapping of their locations in the window was performed to enable ease of locating them afterwards. These two types of microvessels were distinguished on the basis of flow patterns (arterioles: diverging, venules: converging) in their downstream ramifications.

For fluorescence microscopy, a cocktail of the fluorescence compounds $(50 \mu \mathrm{g}$ albumin-FITC [Sigma-Aldrich] $4 \mu \mathrm{g}$ PI [Sigma-Aldrich] and $4 \mu \mathrm{g}$ anti-CD45 PB [Life Technologies, Grand Island, NY, USA]) was intravenously injected through the prewarmed tail vein and allowed to circulate for 15 minutes before imaging was begun. Fluorescence images (320 by 240 pixels) of each vessel were captured using a digital low-light high speed camera (C9300-221; Hamamatsu Photonics, Hamamatsu, Japan) in conjunction with an image acquisition software (HCImage; Hamamatsu Photonics). Green $(518 \mathrm{~nm})$, red $(617 \mathrm{~nm})$ and blue $(460 \mathrm{~nm})$ fluorescence emitted by FITC, PI and PB, respectively were acquired using filter sets (\#41001, \#41002 and \#49021) from Chroma (Rockingham, NY, USA).

For the study of pial arteriolar reactivity, sequential superfusion of the brain cortical surface with test compounds dissolved in aCSF was conducted at a flow rate of $10 \mu \mathrm{L} / \mathrm{min}$ controlled by the syringe pump, while brightfield visualization of the arterioles was carried out based on epiillumination. The height of the outlet tube was adjusted such that intracranial pressure was maintained between 5 and $8 \mathrm{mmHg}$ [21]. All vessel diameter measurements were performed using an image shear device (Image Shear; Vista Electronics, San Diego, CA, USA) with a spatial resolution of $\sim 0.21 \mu \mathrm{m} /$ pixel and images for this purpose were captured by a low light analog camera (COHU 4815; Cohu Electronics, San Diego, CA, USA) and displayed on a color monitor (Pelco, Clovis, CA, USA). MAP was noninvasively acquired using a tail cuff device (CODA system; Kent Scientific, Torrington, CT, USA) both before the start and at the end of the experiment. For velocity measurements in the uninfected mouse, erythrocytes obtained from a donor mouse and fluorescently stained with carbocyanine dye Dil (Molecular Probes, Carlsbad, CA, USA) were used as tracers by intravenous infusion via the tail vein to obtain an in vivo concentration of $\sim 0.4-0.5 \%$ [2]. In the case of the infected mice, $\mathrm{PbA}$ parasitic erythrocytes emitting GFP were utilized as tracers. Video recording of flowing erythrocytes in the vessel was performed at a framing rate of 150 per second for 30 seconds using the high-speed camera in combination with the relevant filter sets (Dil detection: \#41002, GFP detection: \#41001).

\section{Fluorescence Studies on Vascular Leakage, Perivascular Tissue Necrosis, and Leukocyte Adherence}

Analyses on vascular leakage, tissue necrosis, and leukocyte adherence were conducted in the same segment of the vessel for a longitudinal distance of $150 \mu \mathrm{m}$. A comparative study of these parameters was conducted between acute $(n=3)$ and chronic $(n=3)$ window preparations in uninfected animals. The extent of vascular leakage indicates the structural integrity of the blood lumen-vascular tissue interface [28]. An increase in vascular leakage would imply a loss of integrity. On the other hand, the extent of tissue necrosis provides a measure of the degree of tissue damage and inflammation evoked by nonphysiological stimuli (e.g., physical or chemical trauma) [9]. In the present study, vascular leakage was detected by the leakage of infused albumin-FITC from the IV compartment into the surrounding PV tissue, whereas the extent of necrosis in the PV region of the vessel was determined by the intensity of PI stain. A standardized image analysis approach was adopted for quantifications of both leakage and necrosis. The digitalized image file of the vessel labeled by FITC was imported into SigmaScan Pro 4.0 (SPSS, Chicago, IL, USA). The vessel image was first rotated to orientate the vessel longitudinally in a vertical position (see Figure 1H). Luminal vessel wall outlines on opposite sides of the vessel were then manually determined by relying on the image contrast between the bright FITC-fluorescent IV compartment and its surrounding darker, gray background. With reference to these wall outlines, the vessel was divided into IV and PV zones for subsequent analyses in Matlab (R2009a; Mathworks, Natick, MA, USA). In brief, the boundaries of the PV region were defined by translating duplicated outlines in the $x$-direction by distances of $2.6 \mu \mathrm{m}$ (assumed endothelium thickness) and $0.5 \times$ of the vessel diameter (defined PV tissue zone of interest) away from the luminal wall outlines on both sides of the vessel. It should be noted that analysis along the selected vessel segment was conducted on the basis that the PV zone does not contain visible branches of blood flow to minimize background interference from other fluorescent vessels. The digitalized image is composed of pixels with varying light intensity values ranging between 0 (dark pixel) and 1 (bright pixel). Vascular leakage was defined by the ratio between mean pixel intensity values of the PV $\left(I_{\mathrm{PV}}\right)$ and IV $\left(I_{\mathrm{IV}}\right)$ regions (Figure $\left.1 \mathrm{H}\right)$. An increase in this ratio will imply a higher leakage. For necrosis quantification, the wall outlines were superimposed onto corresponding fluorescence image of the same vessel stained by PI. The extent of necrosis in the PV region was subsequently determined by the average pixel intensity in this region (Figure 1I). Necrosis intensity values were normalized to the acute group. A higher intensity value will imply a greater extent of necrosis. Frequency of leukocyte adherence to the vessel wall was used as an indicator of vascular inflammation. Leukocytes were visualized by antiCD45-PB antibodies and adherence was defined as cells that remained static for 30 seconds while in contact with the luminal vessel wall. 


\section{Superfusion Procedures}

Pial arteriolar dilator responses to the applications of endothelium dependent ( $\mathrm{ACh}$; Tocris Bioscience, Minneapolis, MN, USA) and independent (GSNO; Cayman Chemical, Ann Arbor, MI, USA) agonists were compared between acute $(n=5)$ and chronic $(n=5)$ window preparations in uninfected animals. The superfusion protocol was as follows: Baseline diameters of all arterioles were first determined. The arterioles were then suffused with ACh $\left(10^{-5} \mathrm{M}\right)$ for five minutes and their responses were obtained. Following baseline diameter recovery by aCSF washout (10-15 minutes), arteriolar responses to GSNO $\left(10^{-3} \mathrm{M}\right)$ application were tested. Preliminary observations revealed that steady-state dilator responses to the above agonists typically occur within three to five minutes after their introduction.

We further examined changes in pial arteriolar dilator responses during ECM infection. Therefore, comparison of responses was made between ECM $(n=4)$ and uninfected $(n=5)$ cranial window mice prepared using the chronic scheme. The superfusion protocol in these mice was as follows: Baseline arteriolar diameters were first measured and arteriolar responses to five minutes of $\mathrm{ACh}\left(10^{-5} \mathrm{M}\right)$ superfusion were then determined. Next, to study the possible involvement of NO-producing enzymes in the endothelium (e.g., eNOS) in mediating these responses, the cortical surface was suffused with a nonselective NOS inhibitor, L-NMMA $\left(3 \times 10^{-4} \mathrm{M}\right)$ (L-NMMA citrate; Cayman Chemical), for 30 minutes and the arteriolar responses to $\mathrm{ACh}$ were re-examined by the combined superfusion of both ACh and L-NMMA for five minutes. Finally, after the washout of these compounds by aCSF (1015 minutes) and upon recovery of baseline diameters, responses to five minutes of GSNO $\left(10^{-3} \mathrm{M}\right)$ application were tested. In addition to vessel diameter responses, we determined corresponding changes in VFR in 1-2 randomly selected vessels for each preparation. Arteriolar diameter or VFR response was defined as the percent change in vessel diameter or VFR from baseline level after compound superfusion. The chosen concentrations of ACh and L-NMMA followed those reported in previous studies where they were respectively shown to be capable of eliciting prominent pial arteriolar dilation [29] and inhibiting ACh-induced arteriolar dilation [5].

\section{Mean Cellular Velocity and VFR Measurements}

Cellular velocity was measured off-line by first digitalizing the high-speed video recording into its discrete images and then determining the $x-y$ position of a selected erythrocyte in the vessel over consecutive time frames. This procedure was repeated for at least 10 cells at various radial locations in the vessel, and $V$ was obtained by averaging the velocities of all cells. By assuming that the vessel has a cylindrical geometry, VFR in the vessel was approximated using the formula,
VFR $=\Pi(D / 2)^{2} \times V$. All image analyses were performed using the ImageJ software [30].

\section{Parasitological and Clinical Parameters}

Parasitemia of a small blood sample $(\sim 1 \mu \mathrm{L})$ obtained by a mouse tail end prick was determined using flow cytometry by detecting and counting the number of parasitic RBCs that expresses GFP in relation to 10,000 RBCs. Rectal temperature was measured with a thermocouple probe (Oakton $^{\circledR}$ Acorn $^{\text {TM }}$; Oakton Instruments, Vernon Hills, IL, USA) and motor behavioral score was determined by a composite scoring system based on six motor behavioral tests modified from the SHIRPA protocol [17]. ECM was defined as the presentation of one or more of the following clinical signs of neurological involvement: ataxia, limb paralysis, poor righting reflex, seizures, roll-over, and coma.

\section{Statistical Analyses}

All statistical analyses were performed using a statistical software package (Prism 5.0; Graphpad). Paired two-tailed Student's $t$-test was used to determine the statistical significance of effect of L-NMMA inhibition on ACh-induced responses in uninfected and ECM mice. Unpaired two-tailed Student's $t$-test was used to determine the statistical significance of difference in responses between acute and chronic schemes or between uninfected and ECM groups. To compare arteriolar responses among three groups in the same superfusion experiment, a one-way analysis of variance test with Bonferroni post hoc analysis was used. $p<0.05$ was considered statistically significant. All reported data were in mean \pm standard deviation.

\section{RESULTS}

\section{Parasitological and Clinical Parameters}

Parasitemia, rectal temperature, and motor score of mice are shown in Figure $2 \mathrm{~A}-\mathrm{C}$. All parameters were not significantly different between uninfected mice with acute and chronic window preparations (acute: $0.0 \%, 39.0 \pm 0.1^{\circ} \mathrm{C}$ and 23.0, $n=5$; chronic: $0.0 \%, 38.9 \pm 0.2^{\circ} \mathrm{C}$ and $\left.23.0, n=10\right)$. Expectedly, ECM mice developed hypothermia $\left(33.0 \pm 1.7^{\circ} \mathrm{C}, n=4\right)$ with increasing parasitemia $(14.9 \pm 2.6 \%, n=4)$ and lower motor score (9.8 $\pm 2.5, n=4)$ as compared with the uninfected mice, irrespective of the window preparation scheme.

\section{MAP and Arteriolar Diameters}

Using the tail cuff method, MAP measurements in nonanesthetized mice $(110 \pm 6 \mathrm{mmHg}, n=3)$ after recovery from the presurgical procedure were not significantly different from those determined in nonanesthetized healthy mice $(109 \pm 5 \mathrm{mmHg}, n=3)$ that were not subjected to the procedure. In addition, MAP in anesthetized-uninfected cranial window mice before the start of the superfusion 

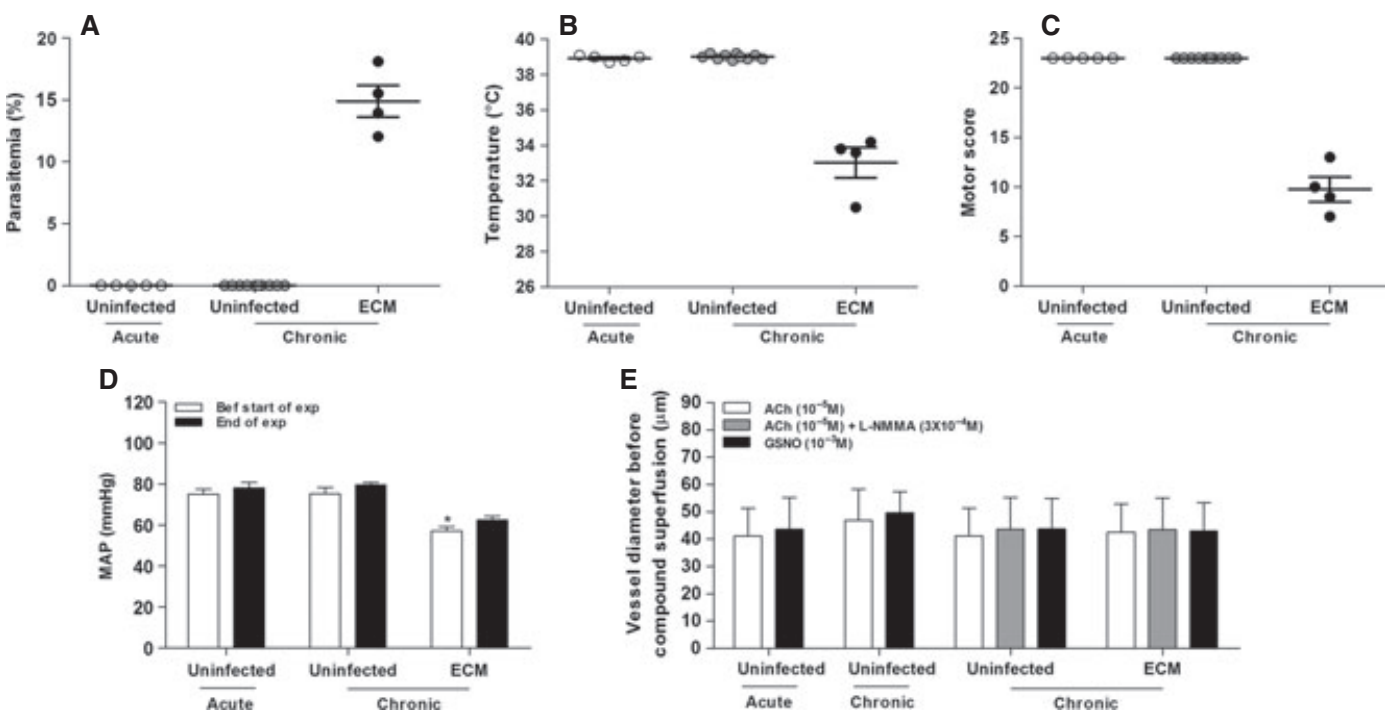

Figure 2. A-C: Parasitemia, rectal temperature and motor behavior score of cranial window mice used for superfusion. (D) MAP in mice before the start and at the end of superfusion experiment. Comparisons were made between uninfected mice with acute and chronic preparations as well as between uninfected and ECM mice with chronic preparations. ${ }^{*} p<0.01$; level of significance compared with uninfected mice with chronic preparations before the start of experiment. (E) Mean arteriolar vessel diameters before the superfusion of compounds in the different groups (ACh and GSNO in both acute and chronic preparations of uninfected mice; ACh, ACh + L-NMMA and GSNO in chronic preparations of both uninfected and ECM mice).

experiment did not significantly vary between chronic (Figure $2 \mathrm{D} ; 75 \pm 3 \mathrm{mmHg}, n=3)$ and acute $(75 \pm 2 \mathrm{mmHg}$, $n=4)$ preparations. These pressure values were also not significantly different from those obtained at the end of the experiment (Figure 2D; chronic: $80 \pm 1 \mathrm{mmHg}, n=3$; acute: $78 \pm 3 \mathrm{mmHg}, n=3$ ) and were consistent with those previously reported by others for anesthetized-healthy cranial window $\mathrm{C} 57 \mathrm{BL} / 6$ mice prepared using the acute scheme $(75 \pm 3 \mathrm{mmHg}$ [29] and $79 \pm 2 \mathrm{mmHg}$ [21]). In contrast, mice with ECM were expected to develop hypotension [10] and in accordance, MAP values of anesthetized-ECM cranial window mice (Figure 2D; $57 \pm 2 \mathrm{mmHg}, n=3$ ) were significantly lower $(p<0.01)$ than those in the anesthetizeduninfected mice, but were similarly not significantly altered at the end of the experiment $(62 \pm 2 \mathrm{mmHg}, n=3)$.

Mean baseline arteriolar diameters of selected vessels for superfusion in all groups did not significantly vary from one another, irrespective of the scheme or the infection status of the mice (Figure 2E; open bars). In addition, mean arteriolar diameters before the superfusion of compounds were not significantly different from the baseline level in all groups (Figure 2E; filled and gray bars).

\section{Comparison of Vascular Leakage, Perivascular Tissue Necrosis, Leukocyte Adherence, and Arteriolar Reactivity Between Acute and Chronic Preparations}

We compared vascular leakage, PV tissue necrosis, and frequency of leukocyte adherence between acute and chronic preparations of uninfected mice. A total of 21 venules (acute:
$D=59.2 \pm 13.6 \mu \mathrm{m}$ and chronic: $D=61.1 \pm 18.6 \mu \mathrm{m})$ and 11 arterioles (acute: $D=60.1 \pm 18.8 \mu \mathrm{m}$ and chronic: $D=64.6 \pm 11.4 \mu \mathrm{m}$ ) were analyzed for each scheme of window preparation. As shown in Figure 3A, vascular leakage was not significantly different between windows prepared by the two schemes, irrespective of the vessel type (arterioles, acute: $0.82 \pm 0.04$ and chronic: $0.82 \pm 0.06$; venules, acute: $0.83 \pm 0.04$ and chronic: $0.83 \pm 0.06)$. These magnitudes of vascular leakage were within the range $(0.5-$ 0.9 ) of those previously reported for classical closed cranial window mice that were adequately recovered from surgery [40]. On the other hand, we found that mean level of tissue necrosis in the arterioles (Figure 3B; acute: $1 \pm 0.82$ and chronic: $0.44 \pm 0.59$ ), but not the venules (acute: $1 \pm 1.45$ and chronic: $0.65 \pm 0.50)$, was significantly reduced $(p<0.05)$ in the chronic as opposed to the acute preparations. No significant difference in frequency of leukocyte adherence to the venular wall was observed between acute and chronic preparations (Figure 3C; acute: $0.44 \pm 0.73$ and chronic: $0.48 \pm 0.59$ ). There was an absence of leukocyte adherence in all arterioles examined regardless of the window preparation scheme.

Arteriolar diameter responses obtained with the acute window preparations were compared against those acquired with the chronic window preparations in uninfected mice (Figure 3D). We found that mean arteriolar dilator responses to both $\mathrm{ACh}(\Delta D=16.4 \pm 8.5 \%, n=19$ arterioles $)$ and GSNO $(\Delta D=26.2 \pm 21.4 \%, n=19)$ applications in the chronic preparations were significantly improved $(p<0.05$ and $<0.01)$ from those $(\mathrm{ACh}: \Delta D=11.1 \pm 8.1 \%$, 

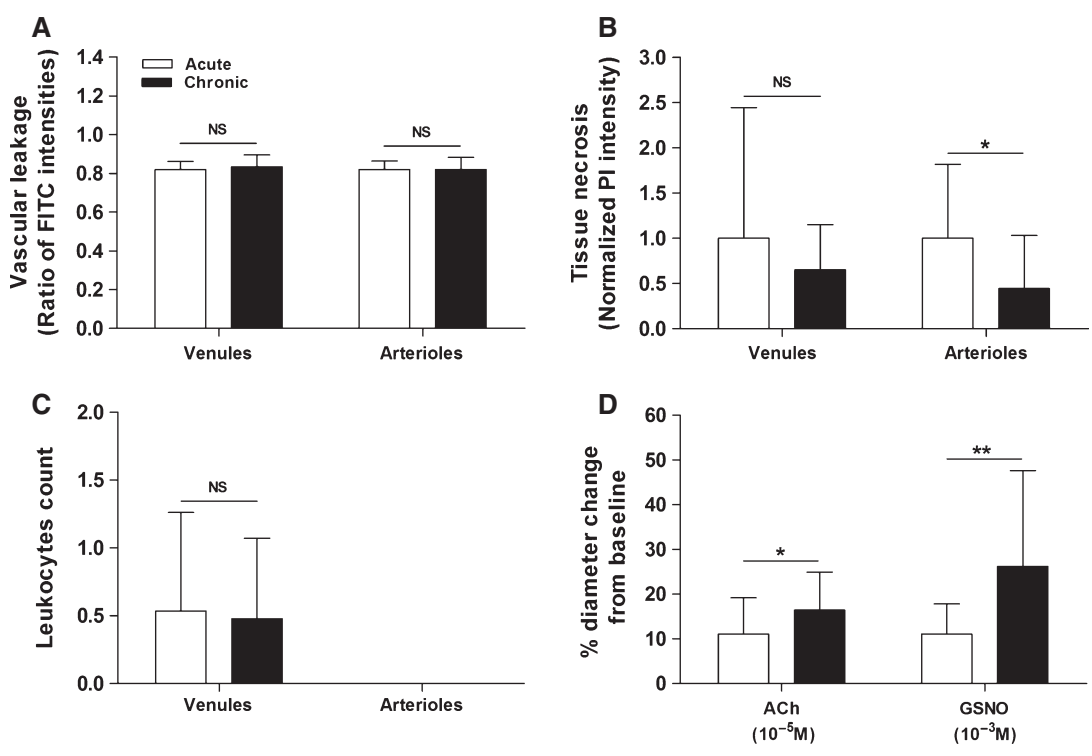

Figure 3. A-C: Comparison of vascular leakage, PV tissue necrosis and frequency of leukocyte adherence between acute and chronic window preparations in uninfected mice. (D) Comparison of arteriolar dilator responses to ACh and GSNO applications between acute and chronic preparations in uninfected mice. ${ }^{*} p<0.05,{ }^{*} p<0.01$, NS, nonsignificant. Data are mean \pm standard deviation.

$n=18$ and GSNO: $\Delta D=11.1 \pm 6.8 \%, n=18)$ in the acute preparations. On closer examination, the overall lower mean magnitude of dilator responses in the acute preparations can be attributed to the considerably small magnitudes $(<6 \%)$ of responses in several arterioles from two of five preparations examined (ACh: 7/18 arterioles and GSNO: 6/18). In contrast, arterioles in all chronic preparations (five of five) consistently produced pronounced dilator responses to the agonists with magnitudes $>9 \%$.

\section{Application of Chronic Scheme to Examine Pial Arteriolar Responses in ECM}

We applied the chronic scheme to investigate changes in both arteriolar diameter and VFR responses during ECM development. Using the chronic window preparation in an ECM mouse, we first demonstrate in Figure 4A apparent widespread vasoconstriction and reduced cerebral blood flow to the brain cortical surface. The preparation remained remarkably stable and did not exhibit any detrimental changes (e.g., bleeding on brain tissue) during superfusion. We then compared arteriolar responses to the applications of $\mathrm{ACh}$, ACh in combination with L-NMMA and GSNO between the uninfected and the ECM mice. Figure 4B shows a typical example of diameter changes in a pial arteriole $(D=43.4 \mu \mathrm{m})$ of an ECM mouse after exposure to ACh and GSNO. The arteriole failed to dilate after ACh treatment, whereas it dilated prominently with a magnitude of $24.2 \%$ when suffused with GSNO. Collectively, we illustrate in Figure $4 \mathrm{C}$ that mean arteriolar dilator response to ACh was significantly attenuated $(p<0.0001)$ by $\sim 90 \%$ in the ECM mice $(\Delta D=1.1 \pm 3.6 \%, n=15$ arterioles $)$ as compared with the uninfected mice $(\Delta D=16.4 \pm 8.5 \%, n=19)$. L-NMMA treatment did not further significantly alter vessel diameter response in the ECM mice $(\Delta D=-0.9 \pm 2.9 \%)$. As arteriolar dilation elicited by ACh treatment in the uninfected mice was significantly diminished $(p<0.0001)$ with L-NMMA treatment, which supported the notion of the involvement of an NOS-dependent mechanism (e.g., NO production by eNOS) in mediating the ACh-elicited dilation, it is possible that eNOS dysfunction could have led to the impaired vascular response in ECM. We also found that overall, arterioles dilated prominently $(\Delta D=16.1 \pm 7.3 \%$, $n=15)$ with GSNO application in the ECM mice although the mean magnitude of this response was $\sim 39 \%$ smaller than that observed in the uninfected mice $(\Delta D=26.2 \pm 21.4 \%$, $n=19)$. This suggested that smooth muscle activity was still intact to some extent in ECM, but pathological events independent of eNOS (e.g., NO quenching by cell-free hemoglobin and reactive oxygen species $[10,37]$ ) may play a role in the mitigation of $\mathrm{NO}$-elicited cerebroarteriolar dilation. As shown in Figure 4D, changes in VFR followed a similar trend to those of vessel diameter. Accordingly, the augmentation of mean VFR $(\triangle \mathrm{VFR}=67.9 \pm 35.3 \%, n=7)$ in the uninfected mice after ACh exposure was significantly decreased $(p<0.05)$ in the ECM mice $(\triangle \mathrm{VFR}=8.2 \pm 36.3 \%$, $n=4$ ), whereas no significant difference in VFR responses was observed in the case of GSNO application. This implied that cerebral blood flow regulation by the eNOS agonist was also impaired in the ECM mice, predominantly due to the loss of pial arteriolar dilator responses. Similar to the vessel 

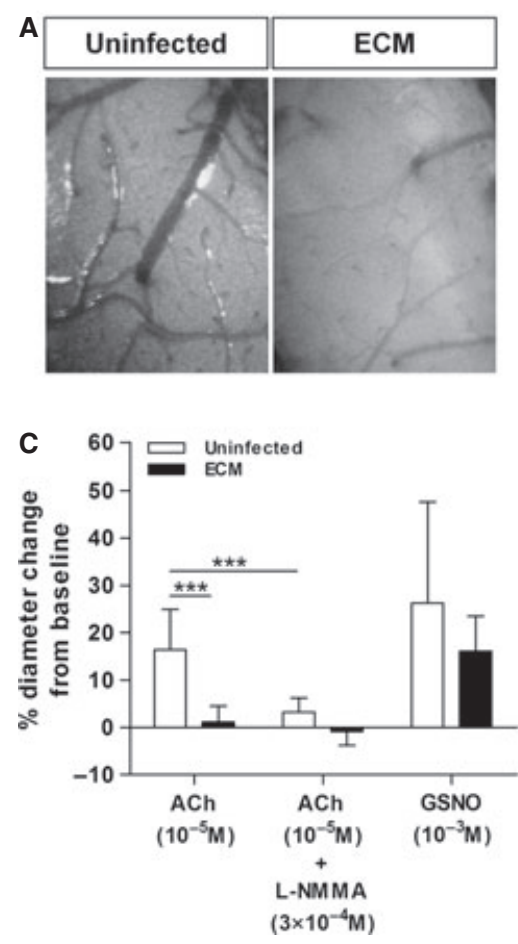
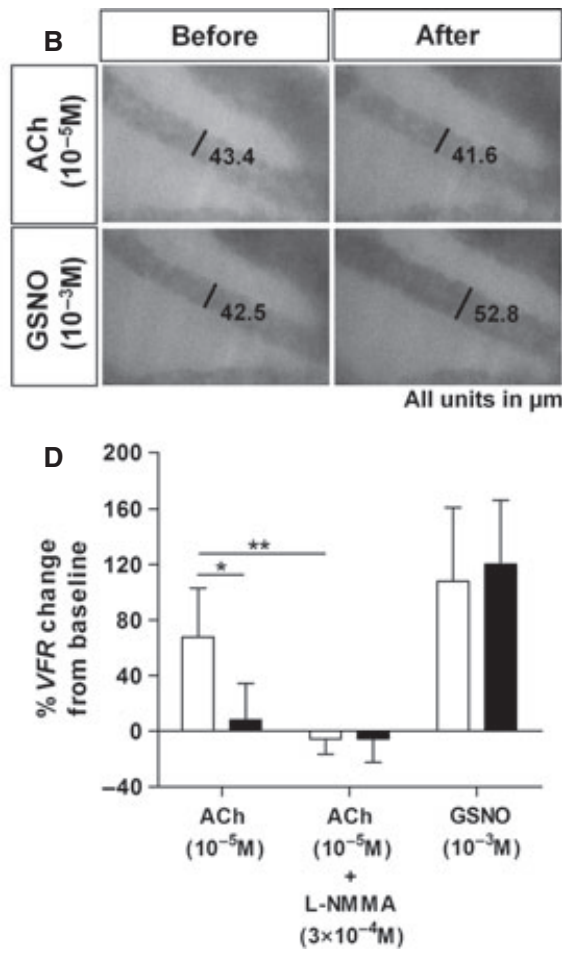

Figure 4. (A) Images on exposed brain cortical surface illustrating apparent vasoconstriction and reduced cerebral perfusion in ECM. (B) Representative images of a pial arteriole in ECM showing changes in its diameter after exposures to ACh and GSNO. (C and D) Comparison of respective mean arteriolar diameter and mean VFR responses between uninfected and ECM mice. ${ }^{*} p<0.05,{ }^{* *} p<0.001,{ }^{* *} p<0.0001$.

diameter responses, L-NMMA treatment abolished $(p<0.001)$ the increase in mean VFR found with ACh application in the uninfected mice.

\section{DISCUSSION}

The concept of cranial superfusion to study pial arteriolar responses in an animal model of human disease through the acute implantation of a closed cranial window is commonly used for enhancing our understanding of cerebrovascular reactivity in many human pathologies (diabetes mellitus [19], obesity [8], hypertension [20], and Alzheimer's disease [27]). Unfortunately, in the execution of an acute scheme, it is inevitable that the animal has to be sequentially subjected to multiple stressful surgical procedures (skin excision $\rightarrow$ skull drilling and removal $\rightarrow$ dura mater removal) for the craniotomy procedure, which limits the applicability of this approach in severely ill animals. In the present study, we adopted a chronic scheme for the window implantation to avoid performing a full craniotomy in the animal when cranial superfusion is needed. By performing the major surgical procedures (skin excision and skull drilling) in the healthy animal beforehand and thereafter introducing a time window (approximately one to two weeks) for postsurgical recovery of the animal, we are able to select animals that survive the surgery and are deemed in good health to undergo the infection or cranial superfusion procedures. This additional step seems pivotal in helping to reduce periarteriolar tissue damage and enhance arteriolar vascular reactivity in the healthy animals, possibly by averting the direct deleterious after effects of mechanical trauma as well as heat-induced blunting of vascular reactivity associated with the skull drilling procedure. Furthermore, our chronic scheme circumvents potentially challenging problems (infection risk and dead space maintenance) associated with dead space formation above the exposed brain cortex in existing chronic superfusion schemes $[15,18]$ by eliminating the need to maintain a preimplanted cranial window over a prolonged period of time. Most importantly, we have demonstrated the feasibility of applying this new scheme to study cerebrovascular reactivity in a severe neuropathology like ECM where the animal's physical condition can precipitously deteriorate within hours and is thus unlikely to bear a full craniotomy procedure. In addition, mice with ECM frequently show brain edema as well as associated coagulopathy, being prone to bleeding. The avoidance of drilling and the gentler removal of the bone flap at the time of experimentation allowed by the new scheme described here resulted in good preservation of the brain tissue and vessels and in rare occurrence of bleeding. In the process, we have shown that this system can be used to unveil mechanisms of cerebrovascular reactivity impairments in sick mice. Indeed, mice 
with ECM were shown to display blunted arteriolar responses to $\mathrm{ACh}$, therefore highlighting a possible role of eNOS dysfunction in ECM development. These preliminary findings validate the application of our chronic scheme to improve our understanding of the mechanistic processes in ECM pathogenesis.

Although demonstrated here in the context of ECM, we would like to emphasize that this new methodology could also appeal to a broader research audience in the study of other infectious encephalopathies with vascular involvement such as sepsis [35] and dengue encephalitis [1]. Alternatively, the new methodology can also be applied in commonly studied diseases in their advanced stages such as stroke [21], as long as a gentler approach of cranial window implantation is warranted to minimize physical stress in the sick animal and to preserve pathologically induced changes in the brain. It should be noted that although the present study was circumscribed to pial arteriolar reactivity (diameters and VFR), this scheme could also be employed for a number of approaches, for instance, the effects of compound superfusion on venules, including diameter and VFR responses, as well as the effects of superfusion on vascular permeability, leukocyte rolling and adhesion during neuroinflammation, and other parameters.

Improving preparation stability is crucial to increase the success rate of experiments involving cranial window superfusion. Indeed, several investigators had reported the need to exclude substandard window preparations from their superfusion experiments, which include preparations that were suffering from apparent signs of instability (e.g., tissue bleeding) [7] or were not exhibiting the anticipated vasodilator responses to the application of agonists [16,38]. Published statistics on experimental success rate are not available in the case of a cranial window preparation for superfusion. Here, we define a successful window preparation in the uninfected animal by its arteriolar responses to ACh where mean magnitude of arteriolar dilation has to be greater than $12 \%$ (equivalent to the lower limit of responses reported in healthy C57BL/6 mice [21]). Therefore, based on this criterion, our present study suggests a preparation success rate of $60 \%$ (three of five mice) in utilization of the acute scheme and $100 \%$ (five of five) in the case of the chronic scheme. It should, however, be cautioned that the robustness of these statistics is limited by the small sample size taken from just our laboratory and that the success rate in the surgery can vary from one operator to another. It is noteworthy that the reported success rate (compiled from five different laboratories) in the classical model of a closed cranial window preparation (e.g., glass coverslip implanted on the exposed cortex) used exclusively for chronic brain imaging is highly variable and operator-dependent, ranging from $30 \%$ to $80 \%$ [11]. We therefore expect the overall success rate to fall in the case of a closed cranial window preparation for superfusion because not only the successful observation of the physiological or pathophysiological phenomena but also the preservation of intrinsic cerebrovascular reactivity after surgical intervention is important in defining a successful preparation.

Underlying causes of experimental failure related to a cranial window preparation are often linked to preparation instability characterized by excessive bleeding from surgery, brain swelling and hemorrhages, and neuroinflammation [38]. It should be emphasized that the occurrence of these symptoms is not spared in chronic schemes, but is usually suppressed through the administration of anti-inflammatory drugs such as dexamethasone (systemic) and epinephrinexylocaine (topical) during and postsurgery [11]. This is possible as the time window for postsurgical animal recovery in the chronic scheme allows the drug effects to adequately subside before commencement of the experiment. In contrast, the acute scheme involves performing experimental observations right after the surgery without recovery of the anesthetized animals. Therefore, the use of these potentially neuromodulating drugs would be impractical due to their confounding effects on brain physiology [13,32] which in turn can complicate experimental outcomes, especially where inflammatory factors are concerned.

Unlike typical superfusion experiments [21,29] in which stability of blood gases at physiological levels in the animal is maintained by mechanical ventilation, this approach was not applied in the present study. This is intended to avoid artificial manipulation of blood gases from pathological levels in ECM mice, which are known to suffer from respiratory distress [10] and to allow an unbiased assessment between findings from uninfected and ECM mice. Nevertheless, several indicators such as the prompt return of the arterioles to their baseline diameters and the minimal perturbations of MAP during the superfusion experiment as well as the reproducible arteriolar responses to agonists (e.g., $10^{-5} \mathrm{M}$ ACh in uninfected mice) of comparable magnitudes with those $(\Delta D \approx 12-20 \%)$ previously reported $[14,29]$ would rule out a major influence of systemic changes in blood gases on arteriolar responses obtained in the present study. It should, however, be noted that the option of ventilation, if required in other circumstances, is perfectly utilizable the same way as it would be with the conventional scheme. Another consideration of the chronic scheme, however, like with the acute scheme, is that the study has to be performed preferably within the shortest time after the protective dura mater layer is breached. Therefore, this scheme does not permit follow-up of the same preparation during the course of PbA infection for instance.

In conclusion, we have developed a new chronic scheme of cranial window preparation suitable for the study of pial arteriolar reactivity in healthy and diseased animal models. This new scheme offers good preparation stability and is especially useful for application in severely ill animals such as 
in ECM where the implementation of a full craniotomy procedure is deemed impractical at the time of experimentation. Therefore, we recommend that the chronic scheme not only can be used in severe cerebrovascular and neurodegenerative diseases, but could become the general method of choice for the study of cerebrovascular reactivity in the living brain.

\section{PERSPECTIVE}

A systematic chronic cranial window preparation scheme for cranial superfusion was adopted for the study of cerebroarteriolar reactivity in living animals, resulting in an improvement in preparation stability. A remarkable advantage of this scheme is its application in severely ill animals, which leads to revelation of novel insights into impaired cerebroarteriolar reactivity and eNOS dysfunction in ECM. This approach has tremendous potential as a research tool to elucidate mechanisms underlying cerebrovascular dysfunction in ECM and possibly other severe cerebrovascular-related pathologies, thereby facilitating the development of new therapeutic strategies to tackle these diseases.

\section{ACKNOWLEDGMENTS}

This work was supported by National Institutes of Health Grants R01-HL87290 and R01-AI82610 (L.J.M.C).

\section{DISCLOSURE/CONFLICT OF INTEREST}

Authors indicated no conflicts of interest.

\section{REFERENCES}

1. Amaral DC, Rachid MA, Vilela MC, Campos $\mathrm{RD}$, Ferreira GP, Rodrigues $\mathrm{DH}$, LacerdaQueiroz N, Miranda AS, Costa VV, Campos MA, Kroon EG, Teixeira MM, Teixeira AL. Intracerebral infection with dengue-3 virus induces meningoencephalitis and behavioral changes that precede lethality in mice. J Neuroinflammation 8: 23, 2011.

2. Cabrales P, Carvalho LJ. Intravital microscopy of the mouse brain microcirculation using a closed cranial window. J Vis Exp 45: e2184, 2010.

3. Cabrales P, Zanini GM, Meays D, Frangos JA, Carvalho LJ. Murine cerebral malaria is associated with a vasospasm-like microcirculatory dysfunction, and survival upon rescue treatment is markedly increased by nimodipine. Am J Pathol 176: 1306-1315, 2010.

4. Capone C, Faraco G, Anrather J, Zhou P, ladecola C. Cyclooxygenase 1-derived prostaglandin E2 and EP1 receptors are required for the cerebrovascular dysfunction induced by angiotensin II. Hypertension 55: 911-917, 2010

5. Crawley DE, Liu SF, Evans TW, Barnes PJ. Inhibitory role of endothelium-derived relaxing factor in rat and human pulmonary arteries. Br J Pharmacol 101: 166170, 1990

6. Desruisseaux MS, Machado FS, Weiss LM, Tanowitz HB, Golightly LM. Cerebral malaria: a vasculopathy. Am J Pathol 176: 1075-1078, 2010.

7. Dirnagl U, Villringer A, Gebhardt R, Haberl RL, Schmiedek P, Einhaupl KM. Threedimensional reconstruction of the rat brain cortical microcirculation in vivo. J Cereb Blood Flow Metab 11: 353-360, 1991.

8. Erdos B, Snipes JA, Miller AW, Busija DW. Cerebrovascular dysfunction in Zucker obese rats is mediated by oxidative stress and protein kinase C. Diabetes 53: 1352 1359, 2004.

9. Fink SL, Cookson BT. Apoptosis, pyroptosis, and necrosis: mechanistic description of dead and dying eukaryotic cells. Infect Immun 73: 1907-1916, 2005.

10. Gramaglia I, Sobolewski P, Meays D, Contreras R, Nolan JP, Frangos JA, Intaglietta $M$, van der Heyde HC. Low nitric oxide bioavailability contributes to the genesis of experimental cerebral malaria. Nat Med 12: 1417-1422, 2006.

11. Holtmaat A, Bonhoeffer T, Chow DK, Chuckowree J, De Paola V, Hofer SB, Hubener M, Keck T, Knott G, Lee WC, Mostany R, Mrsic-Flogel TD, Nedivi E, Portera-Cailliau C, Svoboda K, Trachtenberg JT, Wilbrecht L. Long-term, highresolution imaging in the mouse neocortex through a chronic cranial window. Nat Protoc 4: 1128-1144, 2009.

12. ladecola $C$. Neurovascular regulation in the normal brain and in Alzheimer's disease. Nat Rev Neurosci 5: 347-360, 2004.

13. Ising $M$, Kunzel $H E$, Binder EB, Nickel $T$, Modell S, Holsboer F. The combined dexamethasone/CRH test as a potential surrogate marker in depression. Prog Neuropsychopharmacol Biol Psychiatry 29: 1085-1093, 2005.

14. Kawaguchi T, Brusilow SW, Traystman RJ, Koehler RC. Glutamine-dependent inhibition of pial arteriolar dilation to acetylcholine with and without hyperammonemia in the rat. Am J Physiol Regul Integr Comp Physiol 288: R1612-R1619, 2005.

15. Levasseur JE, Wei EP, Raper AJ, Kontos AA, Patterson JL. Detailed description of a cranial window technique for acute and chronic experiments. Stroke 6: 308-317, 1975.
16. Littleton-Kearney MT, Agnew DM, Traystman RJ, Hurn PD. Effects of estrogen on cerebral blood flow and pial microvasculature in rabbits. Am J Physiol Heart Circ Physiol 279: H1208-H1214, 2000.

17. Martins YC, Smith MJ, Pelajo-Machado M, Werneck GL, Lenzi HL, Daniel-Ribeiro CT, Carvalho LJ. Characterization of cerebral malaria in the outbred Swiss Webster mouse infected by Plasmodium berghei ANKA. Int J Exp Pathol 90: 119-130, 2009.

18. Masuda H, Ushiyama A, Hirota S, Lawlor GF, Ohkubo C. Long-term observation of pial microcirculatory parameters using an implanted cranial window method in the rat. In Vivo 21: 471-479, 2007.

19. Mayhan WG. Responses of cerebral arterioles to activation of beta-adrenergic receptors during diabetes mellitus. Stroke 25: 141-146, 1994.

20. Mayhan WG, Faraci FM, Heistad DD Impairment of endothelium-dependent responses of cerebral arterioles in chronic hypertension. Am J Pathol 253: H1435H1440, 1987.

21. Meng W, Ayata C, Waeber C, Huang PL, Moskowitz MA. Neuronal NOS-cGMPdependent $\mathrm{ACh}$-induced relaxation in pial arterioles of endothelial NOS knockout mice. Am J Pathol 274: H411-H415, 1998.

22. Miller LH, Baruch DI, Marsh K, Doumbo OK. The pathogenic basis of malaria. Nature 415: 673-679, 2002.

23. Morii S, Ngai AC, Winn HR. Reactivity of rat pial arterioles and venules to adenosine and carbon dioxide: with detailed description of the closed cranial window technique in rats. J Cereb Blood Flow Metab 6: 34-41, 1986.

24. Mostany R, Portera-Cailliau C. A craniotomy surgery procedure for chronic brain imaging. J Vis Exp 12: e680, 2008. 
25. Niwa K, Haensel C, Ross ME, ladecola C. Cyclooxygenase- 1 participates in selected vasodilator responses of the cerebral circulation. Circ Res 88: 600-608, 2001.

26. Park L, Anrather J, Zhou P, Frys K, Wang G, ladecola $C$. Exogenous NADPH increases cerebral blood flow through NADPH oxidase-dependent and -independent mechanisms. Arterioscler Thromb Vasc Biol 24: 1860-1865, 2004.

27. Park L, Zhou P, Pitstick R, Capone C, Anrather J, Norris EH, Younkin L, Younkin S, Carlson G, McEwen BS, ladecola C. Nox2-derived radicals contribute to neurovascular and behavioral dysfunction in mice overexpressing the amyloid precursor protein. Proc Natl Acad Sci USA 105: 13471352, 2008.

28. Pino P, Taoufiq Z, Nitcheu J, Vouldoukis I, Mazier D. Blood-brain barrier breakdown during cerebral malaria: suicide or murder? Thromb Haemost 94: 336-340, 2005.

29. Qin X, Kwansa H, Bucci E, Dore S, Boehning D, Shugar D, Koehler RC. Role of heme oxygenase-2 in pial arteriolar response to acetylcholine in mice with and without transfusion of cell-free hemoglobin polymers. Am J Physiol Regul Integr Comp Physiol 295: R498-R504, 2008.
30. Rasband WS, ImageJ, U. S. National Institutes of Health, Bethesda, Maryland, USA, http://rsb.info.nih.gov/ij/, 1997-2005.

31. Reiner G, Clemens R, Bock HL, Enders B. Coagulation disorders in experimentally induced acute mouse malaria. Acta Trop 50: 59-66, 1991.

32. Sinnott CJ, Cogswell IL, Johnson A, Strichartz GR. On the mechanism by which epinephrine potentiates lidocaine's peripheral nerve block. Anesthesiology 98: 181188, 2003.

33. Sobey CG, Moffatt JD, Cocks TM. Evidence for selective effects of chronic hypertension on cerebral artery vasodilatation to protease-activated receptor-2 activation. Stroke 30: 1933-1941, 1999.

34. Sun H, Mayhan WG. Temporal effect of alcohol consumption on reactivity of pial arterioles: role of oxygen radicals. Am J Physiol Heart Circ Physiol 280: H992H1001, 2001.

35. Tore F, Reynier-Rebuffel AM, Tuncel N, Callebert J, Aubineau P. Effects of sepsis on mast cells in rat dura mater: influence of LNAME and VIP. Br J Pharmacol 134: 13671374, 2001.

36. Trauernicht AK, Sun $H$, Patel KP, Mayhan WG. Enalapril prevents impaired nitric oxide synthase-dependent dilatation of cerebral arterioles in diabetic rats. Stroke 34: 2698-2703, 2003.

37. van der Heyde HC, Nolan J, Combes V, Gramaglia I, Grau GE. A unified hypothesis for the genesis of cerebral malaria: sequestration, inflammation and hemostasis leading to microcirculatory dysfunction. Trends Parasitol 22: 503-508, 2006.

38. Xu HT, Pan F, Yang G, Gan WB. Choice of cranial window type for in vivo imaging affects dendritic spine turnover in the cortex. Nat Neurosci 10: 549-551, 2007.

39. Yang G, Pan F, Parkhurst CN, Grutzendler J, Gan WB. Thinned-skull cranial window technique for long-term imaging of the cortex in live mice. Nat Protoc 5: 201-208, 2010.

40. Zanini GM, Cabrales P, Barkho W, Frangos JA, Carvalho LJ. Exogenous nitric oxide decreases brain vascular inflammation, leakage and venular resistance during Plasmodium berghei ANKA infection in mice. J Neuroinflammation 8: 66, 2011. 